\title{
Behavioural contributions to the regulated intake of plant secondary metabolites in koalas
}

\author{
Karen J. Marsh · Ian R. Wallis · William J. Foley
}

Received: 7 February 2007 / Accepted: 17 July 2007 / Published online: 10 August 2007

(C) Springer-Verlag 2007

\begin{abstract}
In a given period of time, herbivores often eat less as dietary plant secondary metabolite (PSM) concentrations increase. This reduction in total food intake is interpreted as a need for the herbivore to regulate PSM ingestion in order to avoid toxication. However, regulation of PSM ingestion involves more than the reduction of total intake; it involves an alteration of meal patterns, through a reduction in the number and/or the size of the meals eaten. Despite this, studies of how herbivores alter their meal patterns when offered varying concentrations of PSMs are rare. We investigated whether koalas adjust the number and/or the size of their meals when offered eucalypt foliage varying naturally in concentrations of formylated phloroglucinol compounds (FPCs), a group of PSMs that have previously been shown to inhibit total food intake. High FPC concentrations caused koalas to eat more slowly, eat shorter meals and eat less per meal, which resulted in a reduced total intake. Because increasing FPC concentrations did not cause koalas to alter the number of meals that they ate, clear individual differences between koalas were observed, where some consistently ate fewer larger meals and others ate many smaller meals. Thus, different feeding strategies may still achieve the same outcome of a regulated intake of PSMs. The changes observed match the meal patterns of other herbivores ingesting PSMs known to stimulate nausea and emetic pathways, supporting the idea that feedback signals from nausea are an important way that koalas avoid toxication when eating eucalypt foliage.
\end{abstract}

Communicated by Ulrich Sommer.

K. J. Marsh ( $₫) \cdot$ I. R. Wallis · W. J. Foley

School of Botany and Zoology, Australian National University,

Canberra, Australian Capital Territory 0200, Australia

e-mail: karen.marsh@anu.edu.au
Keywords Conditioned aversion $\cdot$ Feeding patterns . Formylated phloroglucinol compounds · Herbivore · Nausea

\section{Introduction}

Although many herbivores eat large amounts of a wide variety of plant secondary metabolites (PSMs), few are killed, because they are able to regulate intake to avoid serious harm (Jakubas and Gullion 1990; Provenza et al. 1990; Pfister et al. 1997; Lawler et al. 2000; Stapley et al. 2000). Regulation depends on the herbivores' being able to detect the PSMs in food or to monitor closely the physiological effects of ingested PSMs and to translate them into changes in feeding behaviour (Marsh et al. 2005).

It has been suggested that feedback from PSM ingestion occurs as a common signal that directs feeding and allows herbivores to regulate PSM intake. This common signal is thought to be nauseous malaise (Provenza 1995). Several studies that restore feeding in animals ingesting PSMs, by using drugs to block nausea and emetic pathways, support this theory (Aldrich et al. 1993; Provenza et al. 1994; Lawler et al. 1998).

Any signal providing information about processes that affect total food intake must also affect feeding patterns. For example, Blundell et al. (1985) argued that an animal's response to satiety, nauseous malaise and bitter tastes could be distinguished by studying its meal patterns. They found that satiated rats ate fewer, smaller, meals but ate them at a faster average rate. In contrast, toxins causing nauseous malaise decreased the average rate of feeding and the number of meals, but not the size of meals, while rats responded to a bitter food by eating more, but smaller, meals at a slower average rate (Blundell et al. 1985). 
The vast body of literature on feeding behaviour comes from experiments where researchers exerted tight control by formulating diets. As a consequence, there is scant information on how mammalian herbivores alter their meal patterns when they encounter foods that vary naturally in PSM concentrations (but see Wiggins et al. 2006). Instead, food intake is usually measured over a set time period, often a day or a night, with no effort being made to record the timing and size of individual meals.

To understand better how wild herbivores alter their feeding patterns in response to PSMs, we monitored the feeding patterns of koalas (Phascolarctos cinereus) eating Eucalyptus viminalis foliage. The foliage from individual eucalypts varies naturally in concentrations of formylated phloroglucinol compounds (FPCs), a group of phenolic PSMs whose ingestion is regulated through feedback from the emetic system in other marsupial folivores (Lawler et al. 1998). It is reasonable, therefore, to expect that koalas respond to a similar set of signals, and that these effects would be manifested in their feeding patterns. Whereas the nightly intake of captive koalas declines reliably with increasing concentrations of foliar FPCs (Moore et al. 2005), it is unknown whether they alter the number of meals they eat, the sizes of meals, or both, and whether the behaviour of individuals differs.

\section{Materials and methods}

Six male koalas (mean body mass $\pm \mathrm{SE} 9.2 \pm 0.6 \mathrm{~kg}$ ) were captured on French Island, Victoria, Australia. Female koalas were not used, as most were carrying young at the time. The tooth wear class of each koala was assessed at the time of capture (three class 2 , one 3 , one 4 and one 5), with 0 being the least worn and 7 the most (Martin 1981). The tooth wear class of a koala provides an estimate of their age (Martin 1981), and koalas with worn teeth have previously been shown to spend more time feeding each day than those with less-worn teeth (Logan and Sanson 2002). The koalas were transported to Phillip Island Nature Park and placed individually in pens measuring $2.0 \mathrm{~m} \times 2.5 \mathrm{~m} \times 4.0 \mathrm{~m}$. Each pen contained multiple branches and forks for climbing and sitting, a bowl of water, and four polythene tubes filled with water in which stems of foliage offered as feed were placed. The koalas were allowed to become familiar with captivity over 1 month, during which time they were fed foliage from a variety of eucalypt species (the maintenance foliage).

For the experiment, we randomly allocated 18 Eucalyptus viminalis trees, which were known to vary in FPC concentrations, to three groups. Foliage from each group was fed to koalas following a $6 \times 6$ Latin square design. This resulted in a series of three Latin squares, each experiment lasting 12 days (each experiment day was followed by a rest day, when the koalas were fed the maintenance foliage). One Latin square followed another, so the experiment lasted 35 days. We assessed food intake on one night for each tree for each koala for two reasons. First, koalas (and other eucalypt folivores) have been shown previously to respond quickly to changes in dietary concentrations of FPCs, adjusting their nightly food intake to reflect the concentration of FPCs in the food offered to them on that night (Lawler et al. 2000; Stapley et al. 2000; Moore et al. 2005). Thus, we anticipated that a single night would show us how a koala would respond if it encountered each tree in the wild. Second, koalas eat little when offered foliage with medium-to-high concentrations of FPCs, and we did not want to deprive them of food for more than a single night.

The branches of experimental foliage were collected the day before each period started and consisted of mature foliage only, as there may be differences in FPC concentration between young and old foliage from the same tree (Moore 2004). In contrast, there is little variation in FPC concentrations between branches of mature foliage within a tree (K. Marsh, unpublished data). Branches were stored with the cut stems in water at $4^{\circ} \mathrm{C}$. At 1400 hours on experiment days, we removed the maintenance foliage and then, at 1800 hours, offered the koalas branches of experimental foliage. Additional branches were placed outside the pens so that we could monitor changes in weight in the absence of herbivory. Using a torch fitted with a red filter, we observed the koalas from 1800 hours until 0600 hours the following morning. We recorded the time that a koala began to feed and when it stopped. Ten minutes after feeding had ended, indicating the end of a meal, we removed, weighed and replaced the branches. These measurements allowed us to calculate the time and duration of meals and intervals between them, the number of meals eaten, the amount of foliage eaten in each meal, and the feeding rate. We also estimated the time it took the koalas to eat $50 \%$ of their nightly intake, to gauge shifts in the timing of feeding. At 0600 hours, all branches were removed, weighed and replaced with fresh branches of maintenance foliage. As weight changes in the control branches were never more than $0.5 \%$, no corrections were made for water loss. We weighed the koalas weekly, to ensure that they were maintaining body mass. Samples of all foliage offered were dried at $60^{\circ} \mathrm{C}$ to determine the dry matter (DM) content and, hence, to calculate dry matter intake (DMI). Additional samples were frozen at $-20^{\circ} \mathrm{C}$ for later analysis for FPCs and cyanogenic glycosides.

\section{Determination of FPC concentrations}

Leaf samples were freeze-dried and ground in a Cyclotec 1093 Mill (Tecator, Hoganas, Sweden) to pass through a 
$1 \mathrm{~mm}$-mesh sieve. The ground samples were stored in plastic vials in the dark at room temperature. FPCs were extracted and then analysed by high performance liquid chromatography (HPLC) as described in Moore et al. (2004). Because different FPC types appear to have a similar effect on koala feeding (Moore et al. 2005), we added the individual FPC concentrations together to give a value that we called "total FPCs".

Presence/absence of cyanogenic glycosides

Freeze-dried leaves were crushed into glass vials and moistened with $200 \mu \mathrm{l}$ phosphate buffer (pH 5.0, $0.1 \mathrm{M}$ ), or $200 \mu \mathrm{l}$ phosphate buffer containing $\beta$-glucosidase extracted from almonds (1.12 units $\mathrm{ml}^{-1}$; Sigma). A strip of Feigl-Anger paper was suspended in each vial, and the vials were capped with rubber stoppers. The colour of the paper was recorded after $24 \mathrm{~h}$. Crushed apple pips were used as a positive control for the presence of cyanogenic glycosides.

\section{Statistical analysis}

Although our main question was how the concentration of FPCs in foliage affected various aspects of feeding behaviour, other factors such as the tooth wear class and body mass of the koalas, the time of night at which a meal was eaten, the length of time that had passed since the previous meal, the amount eaten in the previous meal, the number of meals eaten in a night, and interactions between these terms could also potentially influence feeding. Thus, we used the residual maximum likelihood (REML) algorithm in Genstat 7.1 (Numerical Algorithms Group, Oxford, UK) for statistical analyses, because it allowed us to investigate the effects of all these factors simultaneously. In each model, all of the relevant identifiable sources of variation from the list above were included as fixed effects, and non-significant terms were sequentially removed from full models to create final models with only significant effects $(P<0.05)$. Individual koalas were included as random effects in models unless we were specifically interested in how individual variation affected the response variate (i.e. whether individual koalas ate different numbers of meals and had different average FPC intakes). Thus, if we describe differences between individual koalas, these results were obtained when the koalas were included as fixed effects in the model. The day on which measurements were made was always included as a random effect. Reported $P$ values are from the full model if nonsignificant $(P>0.05)$ and from the reduced model if significant. All data were checked for normality and were $\log$ transformed when necessary.

\section{Results}

Neither tooth wear class nor body mass affected any of the variables measured $(P>0.05$ in all tests), and so they are not reported again in the results.

Variation in FPC and cyanide content of foliage offered to koalas

The foliage from the 18 Eucalyptus viminalis trees offered to the koalas varied widely in the concentration of individual and total FPCs. Most trees were dominated by "group 1" macrocarpals (macrocarpals G, A, B, eucalyptone, and those that elute at $47 \mathrm{~min}, 39 \mathrm{~min}, 35 \mathrm{~min}$ and 27 min; Moore et al. 2004). "Group 2" macrocarpals (macrocarpals $\mathrm{I}$ and $\mathrm{J}$, and those that elute at $42 \mathrm{~min}$ and $34 \mathrm{~min}$; Moore et al. 2004) were found in only one tree. Sideroxylonals $\mathrm{A}$ and $\mathrm{C}$ were present in most trees. In keeping with previous results (Moore et al. 2005), the koalas did not treat the tree containing group 2 macrocarpals (total concentration $30.1 \mathrm{mg} \mathrm{g}^{-1} \mathrm{DM}$ ) any differently from those with group 1 macrocarpals (total concentration ranging between $4.4 \mathrm{mg} \mathrm{g}^{-1}$ and $48.7 \mathrm{mg} \mathrm{g}^{-1} \mathrm{DM}$; see figures), so a measure of "total FPCs" appeared to be valid. None of the foliage offered to the koalas contained cyanogenic glycosides.

What influences DMI and the amount of time koalas spend feeding?

Each night, the six koalas spent anywhere between $10 \mathrm{~min}$ and $5 \mathrm{~h}$ (mean $\pm \mathrm{SE}=128 \pm 6 \mathrm{~min}$ ) feeding, and they ate between $9 \mathrm{~g}$ and $314 \mathrm{~g}$ DM (mean $\pm \mathrm{SE}=146 \pm 7 \mathrm{~g}$ ). There were strong correlations between the amount eaten and the time the koalas spent feeding in a night $(P<0.001$; $\left.r^{2}=0.79\right)$ and per meal $\left(P<0.001 ; r^{2}=0.86\right)$. Thus, analyses based on either the time spent feeding, or on the amount eaten, gave similar results. The koalas ate less overall $(P<0.001$; Fig. 1a), fed for a shorter time $(P<0.001)$, ate less per meal $(P<0.001$; Fig. $1 \mathrm{~b})$ and had shorter meals $(P<0.001)$ as FPC concentrations in foliage increased. A significant interaction effect between the number of meals eaten by the koalas and the FPC concentration of foliage revealed that no matter whether the koalas chose to eat many small, or a few large, meals, they ate the same amount of foliage at the lowest FPC concentrations. In contrast, as FPC concentrations increased, the feeding strategy adopted by individual koalas affected their food intake, so that at the highest FPC concentrations, koalas that ate many small meals were able to eat about three times as much as koalas that ate a few larger meals $(P=0.05)$. This is further illustrated by the fact that coefficients of variation $(\mathrm{CVs})$ were much higher (55-67) when the koalas ate foliage from 

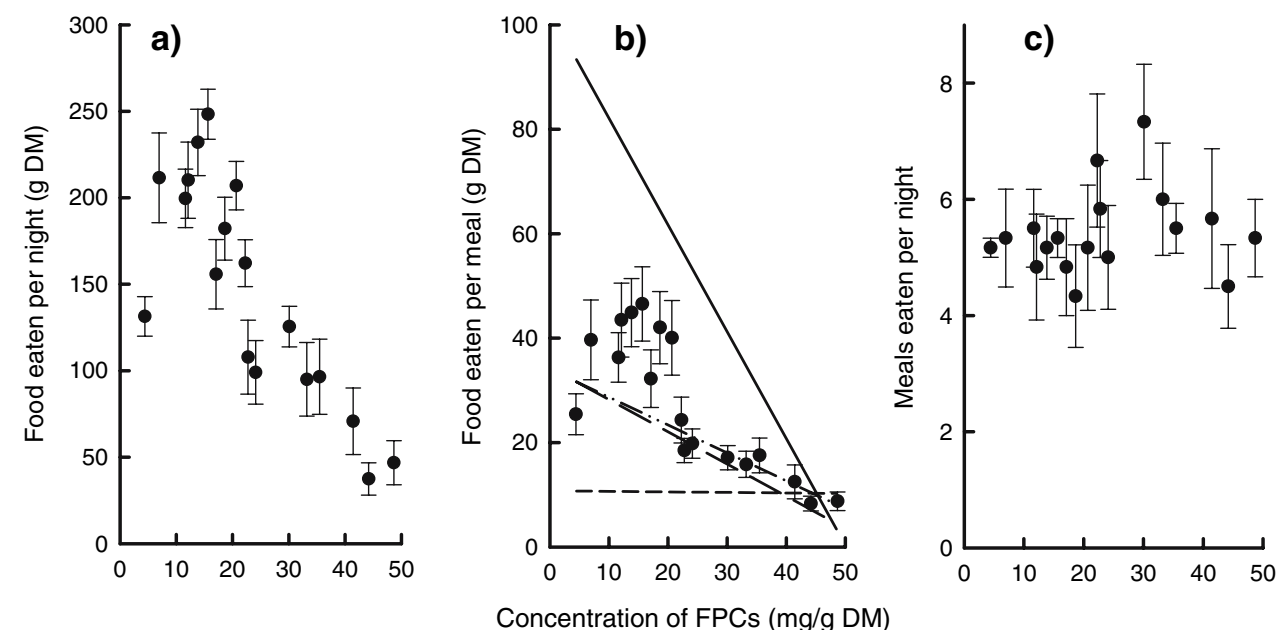

Fig. 1 The responses of six koalas to varying FPC concentrations in Eucalyptus viminalis foliage. a The amount of foliage eaten over $12 \mathrm{~h}$ of ad libitum presentation (mean \pm SE). $\mathbf{b}$ The amount of foliage eaten during each meal (circles; mean $\pm \mathrm{SE}$ ). The lines are a summary of the factors identified by REML modelling that had a significant effect on the amount of food eaten by the koalas during a meal, namely the time of night, the FPC concentration of foliage, and the number of meals initiated during the night (solid line 1800 hours, four meals; (long dashed line 1800 hours, eight meals; (alternating dashed and dotted line 0400 hours, four meals; (short dashed line 0400 hours, eight meals). c The number of meals eaten per night by the koalas $($ mean $\pm \mathrm{SE})$

Table 1 A summary of the feeding behaviour of individual koalas, averaged across 18 nights (mean \pm SE)

\begin{tabular}{llll}
\hline Koala & $\begin{array}{l}\text { Meals } \\
\text { per night }\end{array}$ & $\begin{array}{l}\text { FPCs ingested } \\
\text { per night }(\mathrm{mg})\end{array}$ & $\begin{array}{l}\text { FPCs ingested } \\
\text { per meal }(\mathrm{mg})\end{array}$ \\
\hline 1 & $7.4 \pm 0.4^{\mathrm{a}}$ & $2,291 \pm 214^{\mathrm{a}}$ & $310 \pm 25^{\mathrm{a}}$ \\
2 & $5.1 \pm 0.3^{\mathrm{b}}$ & $2,606 \pm 262^{\mathrm{a}, \mathrm{b}}$ & $510 \pm 39^{\mathrm{b}}$ \\
3 & $4.9 \pm 0.5^{\mathrm{b}, \mathrm{c}}$ & $2,311 \pm 336^{\mathrm{a}}$ & $468 \pm 59^{\mathrm{b}}$ \\
4 & $5.1 \pm 0.4^{\mathrm{b}}$ & $2,982 \pm 329^{\mathrm{b}, \mathrm{c}}$ & $590 \pm 60^{\mathrm{b}, \mathrm{c}}$ \\
5 & $4.2 \pm 0.3^{\mathrm{c}}$ & $3,221 \pm 417^{\mathrm{b}, \mathrm{c}}$ & $773 \pm 102^{\mathrm{c}}$ \\
6 & $5.8 \pm 0.5^{\mathrm{b}}$ & $3,253 \pm 268^{\mathrm{c}}$ & $558 \pm 45^{\mathrm{b}}$ \\
\hline
\end{tabular}

Values in a column that share the same letter are not significantly different from one another

koalas slowing slightly, but significantly, with increasing concentrations of FPCs $(P=0.01)$. Thus, the koalas fed $0.1 \mathrm{~g} \mathrm{~min}^{-1}$ slower on the foliage with the highest concentration of FPCs, relative to the lowest.

Do FPCs affect the amount of time koalas take to eat $50 \%$ of their nightly intake?

Even though the koalas ate less of the foliage with high FPC concentrations, it took them longer to eat half of their total nightly intake $(P<0.001)$. Koalas offered the foliage with the lowest concentration of FPCs had eaten roughly half of their nightly intake by 2200 hours, $4 \mathrm{~h}$ into the $12 \mathrm{~h}$ feeding period. In contrast, koalas offered the foliage with the highest FPC concentration took almost $7 \mathrm{~h}$ to eat half of their food. 
Do FPCs cause koalas to alter the lengths of non-feeding intervals between meals?

The non-feeding intervals between meals varied in length between $10 \mathrm{~min}$ and $470 \mathrm{~min}$, but they were not influenced by the concentration of FPCs in foliage $(P=0.86)$, or by the amount of food or FPCs ingested during the previous meal $(P=0.81$ and $P=0.18$, respectively). Instead, both the time of night and the number of meals eaten by koalas affected non-feeding intervals. More specifically, as the night progressed, the intervals between meals increased in length by between $50 \mathrm{~min}$ and $90 \mathrm{~min}$, regardless of the FPC concentration $(P<0.001)$, while non-feeding intervals were shorter for the koalas that ate many meals, compared to those that ate few $(P<0.001)$.

\section{What factors affect FPC intake by koalas?}

The amount of FPCs that the koalas ingested during a night increased with FPC concentrations of up to $21 \mathrm{mg} \mathrm{g}^{-1} \mathrm{DM}$ $(P<0.001)$. At FPC concentrations higher than this, FPC intake appeared to decline, but a simple regression analysis showed that there was no difference in FPC intake from concentrations of $21-50 \mathrm{mg} \mathrm{g}^{-1} \mathrm{DM}$ ( $P=0.16$; Fig. $\left.2 \mathrm{a}\right)$. When individual koalas were included as a fixed effect in a
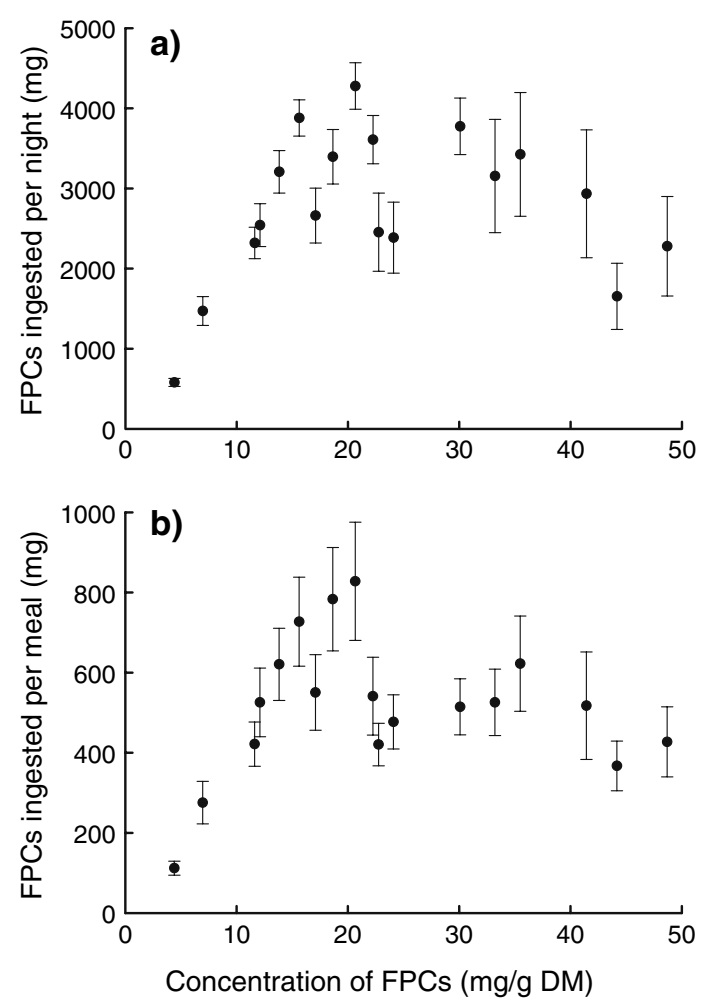

Fig. 2 The average (a) nightly intake and (b) intake per meal of FPCs (mean \pm SE) by six koalas offered foliage containing different FPC concentrations
REML analysis, the average nightly intake of FPCs was higher for some individuals than others $(P<0.01$; Table 1$)$.

The intake of FPCs per meal showed a similar pattern to nightly FPC intake. In other words, regression analysis showed that FPC intake increased with FPC concentrations up to $21 \mathrm{mg} \mathrm{g}^{-1} \mathrm{DM}(P<0.001)$, but not above this $(P=0.58$; Fig. 2b). REML analysis revealed that more FPCs were ingested during a meal when the pre-meal interval was longer $(P<0.001)$ and when few meals were eaten $(P=0.023)$. Also, when included as fixed effects, some individual koalas ingested more FPCs during each meal than others $(P<0.001$; Table 1$)$.

\section{Discussion}

Our study shows that behavioural modifications of meal size, but not meal frequency, play a vital role in allowing koalas to regulate their intake of plant secondary metabolites (PSMs) in Eucalyptus foliage. Koalas responded to increasing concentrations of FPCs by eating less per meal, feeding for less time and at a slower average rate, without altering the number of meals that they ate. By eating the same number of meals at all FPC concentrations, the koalas exhibited a variety of feeding strategies that were specific to individual koalas. In particular, some koalas consistently ate few meals, while others ate many. Behaviour is flexible and is influenced by past experiences (Distel and Provenza 1991; Villalba and Provenza 2000; Provenza et al. 2003; Villalba et al. 2004), so it is unrealistic to expect all individuals to conform to a single behavioural pattern. However, in the presence of PSMs that may potentially have toxic effects if over ingested, it is interesting that the different feeding strategies still achieved regulated PSM intake without the animals being poisoned.

The willingness of the koalas to alter meal size, but not meal frequency, led to several interesting outcomes. The first was that, at low FPC concentrations, the two feeding strategies: few large meals, or many small meals, did not make a difference to food intake. However, the koalas that ate many small meals ingested up to three-times more food at high FPC concentrations and, therefore, met a larger proportion of their daily intake requirements. Thus, at high FPC concentrations, the individual koalas that ate many small meals were at an advantage. This raises the question of why the other koalas did not actively compensate for increasing FPC concentrations by eating more meals. It may have been that the feeding pattern demonstrated by each individual koala was the result of its own previous experience of foraging in the wild. Wild koalas probably rarely need to adjust their feeding behaviour to increase intake, because food is not limiting. It is likely that they inhabit home ranges that contain trees covering the 
spectrum of FPC concentrations (Lawler et al. 2000; Wallis et al. 2002), so that they normally have the option of avoiding less palatable trees or of following a meal of less palatable foliage with one of palatable foliage. In this situation, it is likely that the different feeding strategies demonstrated by the captive koalas would be equally viable. It would be interesting to test in captivity whether individuals could learn to shift their feeding strategy over time if they were not given palatable food in the morning.

The second outcome was that individual koalas that ate few meals often ingested more than $1 \mathrm{~g}$ FPCs per meal, while those that fed more regularly rarely exceeded this amount. However, because koalas that ate few meals also spaced them further apart, they had longer to detoxify the ingested FPCs before the next meal, whereas, koalas that ate many meals had little time for detoxification. This may reflect different tolerance levels and detoxification rates for FPCs between individuals, and it would be interesting to keep these differences in mind if future studies were to compare detoxification thresholds and rates with feeding patterns.

The meal pattern changes demonstrated by the koalas in this study match those of other mammals voluntarily ingesting PSMs. More specifically, rats fed the cyanoglycoside, simmondsin (Lievens et al. 2003), brushtail possums fed the terpene, 1,8-cineole (Wiggins et al. 2003), desert woodrats fed phenolic resin from creosote bush (Sorensen et al. 2005) and ringtail possums fed foliage containing FPCs (Wiggins et al. 2006), all ate smaller and slower meals. That final study is of particular relevance to ours, because ringtail possums are known to regulate FPC intake via feedback from the emetic system (Lawler et al. 1998). Blundell et al. (1985) suggested that different feedback signals would be manifested as changes in different aspects of feeding patterns and showed that satiety, nauseous malaise, and bitter tastes produced different feeding responses in rats. Thus, the similar feeding patterns demonstrated by five different species of animals fed diets containing PSMs may illustrate that nausea and emesis can act as a common signal to regulate PSM ingestion.

However, if nausea and emesis provide a key signal to herbivores that regulate their intake of PSMs, explanation is required for why their feeding patterns may differ from those traditionally associated with nauseous malaise. In other words, rats experiencing malaise from an injection of lithium chloride $(\mathrm{LiCl})$, decreased their average rate of feeding and the number of meals that they ate, but they maintained the size of the meals (Blundell et al. 1985; West et al. 1987). The different behavioural responses of animals regulating PSM intake, compared with rats injected with $\mathrm{LiCl}$, is probably a consequence of the multiple signals received by an animal confronted with foods containing PSMs. The palatability of foods eaten by wild herbivores may depend on competition between the need for nutrients and avoidance of toxicity (Marsh et al. 2006). Furthermore, previous experience with a PSM allows animals to anticipate the consequences of ingestion (Distel and Provenza 1991; Villalba and Provenza 2000; Provenza et al. 2003; Villalba et al. 2004), which can alter feeding behaviour (Lievens et al. 2003). Animals learn to associate the flavour of a food with its post-ingestive consequences, and when flavour cues reliably indicate toxin concentrations, they can regulate intake based on the strength of the flavour alone (Launchbaugh et al. 1993; Lawler et al. 1999).

Physiological limitations to detoxification may also cause different feeding responses between studies that inject animals with the test compound and those that allow them to eat freely. Herbivores are poisoned if they ingest PSMs faster than they are able to detoxify them (Freeland and Janzen 1974; Pfister et al. 1997; Galey et al. 1998; Marsh et al. 2005). Rats injected with $\mathrm{LiCl}$ and offered a palatable food will not subsequently ingest too much of the toxin. In contrast, the concentration of a PSM in food places an upper limit on the rate of PSM ingestion and, therefore, how much of that food a herbivore can eat (Marsh et al. 2005). As a consequence, it may not be possible for a free-feeding herbivore, unlike rats injected with a toxin like $\mathrm{LiCl}$, to maintain the length of a meal when ingesting a PSM, without increasing the risk of being poisoned.

This idea is supported by the fact that FPCs were the only significant factor affecting the size of meals at high FPC concentrations, whereas the time of night and the number of meals a koala ate also played a role at low concentrations. As a result, koalas ate similar sized meals throughout the night at high FPC concentrations and, therefore, took approximately half of the night to eat half of their food. Because the koalas ate larger meals at the start than at the end of the night at low FPC concentrations, they took only $4 \mathrm{~h}$ to eat the first half of their food, but $8 \mathrm{~h}$ for the second. Thus, there was a shift in the timing of feeding across the FPC concentrations. There are also likely to be effects of other PSMs and nutrients in the leaves on feeding at low FPC concentrations that we did not measure, which is unavoidable in studies conducted with natural diets. Perhaps the influence of other unmeasured foliar components would explain why the koalas found the foliage that contained the lowest concentration of FPCs $\left(4.4 \mathrm{mg} \mathrm{g}^{-1} \mathrm{DM}\right)$ less palatable than some that had higher FPC concentrations (Fig. 1a, b).

Taken together, our results suggest that FPCs are likely to affect the feeding decisions of free-ranging koalas. Essentially, koalas utilising trees with high concentrations of FPCs need to tolerate smaller meals, or increase their intake in subsequent meals by seeking trees with lower FPC concentrations. Reduced intake is an important cost of PSM 
ingestion, because, as food intake falls, so too does intake of nutrients, especially when eucalypt foliage is a poor source of nutrients anyway (Cork 1996). Some cohorts of koalas that require extra food, including lactating animals (Krockenberger 2003; Logan and Sanson 2003), those with worn teeth (Logan and Sanson 2002), and those in cold conditions (Harrop and Degabriele 1976; Ellis et al. 1995), might not be able to achieve this without specifically selecting trees that allow them to eat more. We propose that freeranging koalas, intent on feeding, should rarely enter trees that contain high concentrations of FPCs. If they do they should stay for a short period of time and eat small meals.

Acknowledgements Dr. Kath Handasyde, Dr. Ben Moore and Parks Victoria staff assisted with the capture and release of the koalas. Phillip Island Nature Park, and Marg Healy, in particular, kindly allowed us to use their koala pens and eucalypt plantation. Thanks also to Linda Merrin, Owen and Jan Marsh, and Cora Shiroyama, who helped with some of the care of the koalas. Dr. Ann Cowling and Ms. Christine Donnelly from the Statistical Consulting Unit at the Australian National University gave advice on statistical analyses. This research was approved by the Victorian Department of Sustainability and Environment and the Animal Experimentation Ethics Committee of the Australian National University and conforms to the Australian Code of Practice for the Care and Use of Animals for Scientific Purposes.

\section{References}

Aldrich CG, Rhodes MT, Miner JL, Kerley MS, Paterson JA (1993) The effects of endophyte-infected tall fescue consumption and use of a dopamine antagonist on intake, digestibility, body temperature, and blood constituents in sheep. Anim Sci 71:158-163

Blundell JE, Rogers PJ, Hill AJ (1985) Behavioural structure and mechanisms of anorexia: calibration of natural and abnormal inhibition of eating. Brain Res Bull 15:371-376

Cork SJ (1996) Optimal strategies for arboreal herbivorous mammals in contrasting forest types: why koalas and colobines are different. Aust J Ecol 21:10-20

Distel RA, Provenza FD (1991) Experience early in life affects voluntary intake of blackbrush by goats. J Chem Ecol 17:421-450

Ellis WAH, Melzer A, Green B, Newgrain K, Hindell MA, Carrick FN (1995) Seasonal variation in water flux, field metabolic rate and food consumption of free-ranging koalas (Phascolarctos cinere$u s)$. Aust J Zool 43:59-68

Freeland WJ, Janzen DH (1974) Strategies in herbivory by mammals: the role of plant secondary compounds. Am Nat 108:269-289

Galey FD, Holstege DM, Johnson BJ, Siemens L (1998) Toxicity and diagnosis of oleander (Nerium oleander) poisoning in livestock. In: Garland T, Barr AC (eds) Toxic plants and other natural toxicants. CAB International, Oxon, pp 215-219

Harrop CJF, Degabriele R (1976) Digestion and nitrogen metabolism in the koala, Phascolarctos cinereus. Aust J Zool 24:201-215

Jakubas WJ, Gullion GW (1990) Coniferyl benzoate in quaking aspen-a ruffed grouse feeding deterrent. J Chem Ecol 16:10771087

Krockenberger A (2003) Meeting the energy demands of reproduction in female koalas, Phascolarctos cinereus: evidence for energetic compensation. J Comp Physiol B 173:531-540

Launchbaugh KL, Provenza FD, Burritt EA (1993) How herbivores track variable environments: response to variability of phytotoxins. J Chem Ecol 19:1047-1056
Lawler IR, Foley WJ, Pass GJ, Eschler BM (1998) Administration of a $5 \mathrm{HT}_{3}$ receptor antagonist increases the intake of diets containing Eucalyptus secondary metabolites by marsupials. J Comp Physiol B 168:611-618

Lawler IR, Stapley J, Foley WJ, Eschler BM (1999) Ecological example of conditioned flavor aversion in plant-herbivore interactions: effect of terpenes of Eucalyptus leaves on feeding by common ringtail and brushtail possums. J Chem Ecol 25:401-415

Lawler IR, Foley WJ, Eschler BM (2000) Foliar concentration of a single toxin creates habitat patchiness for a marsupial folivore. Ecology $81: 1327-1338$

Lievens S, Flo G, Decuypere E, Van Boven M, Cokelaere M (2003) Simmondsin: effects on meal patterns and choice behavior in rats. Physiol Behav 78:669-677

Logan M, Sanson GD (2002) The effect of tooth wear on the feeding behaviour of free-ranging koalas (Phascolarctos cinereus, Goldfuss). J Zool 256:63-69

Logan M, Sanson GD (2003) The effects of lactation on the feeding behaviour and activity patterns of free-ranging female koalas (Phascolarctos cinereus Goldfuss). Aust J Zool 51:415-428

Marsh KJ, Wallis IR, Foley W (2005) Detoxification rates constrain feeding in common brushtail possums (Trichosurus vulpecula). Ecology 86:2946-2954

Marsh KJ, Wallis IR, McLean S, Sorensen JS, Foley WJ (2006) Conflicting demands on detoxification pathways influence how brushtail possums choose their diets. Ecology 87:2103-2112

Martin RW (1981) Age-specific fertility in three populations of koala, Phascolarctos cinereus (Goldfuss), in Victoria. Aust Wildl Res 8:275-283

Moore BD (2004) Chemical determinants of diet and habitat quality in the koala Phascolarctos cinereus Goldfuss. PhD thesis. Australian National University, Canberra

Moore BD, Wallis IR, Palá-Paúl J, Brophy JJ, Willis RH, Foley WJ (2004) Antiherbivore chemistry of Eucalyptus - cues and deterrents for marsupial folivores. J Chem Ecol 30:1743-1769

Moore BD, Foley WJ, Wallis IR, Cowling A, Handasyde KA (2005) Eucalyptus foliar chemistry explains selective feeding by koalas. Biol Lett 1:64-67

Pfister JA, Provenza FD, Manners GD, Gardner DR, Ralphs MH (1997) Tall larkspur ingestion: can cattle regulate intake below toxic levels? J Chem Ecol 23:759-777

Provenza FD (1995) Postingestive feedback as an elementary determinant of food preference and intake in ruminants. J Range Manage 48:2-17

Provenza FD, Burritt EA, Clausen TP, Bryant JP, Reichardt PB, Distel RA (1990) Conditioned flavor aversion: a mechanism for goats to avoid condensed tannins in blackbrush. Am Nat 136:810-828

Provenza FD, Ortegareyes L, Scott CB, Lynch JJ, Burritt EA (1994) Antiemetic drugs attenuate food aversions in sheep. J Anim Sci 72:1989-1994

Provenza FD, Villalba JJ, Dziba LE, Atwood SB, Banner RE (2003) Linking herbivore experience, varied diets, and plant biochemical diversity. Small Rumin Res 49:257-274

Sorensen JS, Heward E, Dearing MD (2005) Plant secondary metabolites alter the feeding patterns of a mammalian herbivore (Neotoma lepida). Oecologia 146:415-422

Stapley J, Foley WJ, Cunningham R, Eschler B (2000) How well can common brushtail possums regulate their intake of Eucalyptus toxins? J Comp Physiol B 170:211-218

Villalba JJ, Provenza FD (2000) Roles of flavor and reward intensities in acquisition and generalization of food preferences: do strong plant signals always deter herbivory? J Chem Ecol 26:1911-1922

Villalba JJ, Provenza FD, Han G-D (2004) Experience influences diet mixing by herbivores: implications for plant biochemical diversity. Oikos 107:100-109 
Wallis IR, Watson ML, Foley WJ (2002) Secondary metabolites in Eucalyptus melliodora: field distribution and laboratory feeding choices by a generalist herbivore, the common brushtail possum. Aust J Zool 50:1-13

West DB, Greenwood MRC, Marshall KA, Woods SC (1987) Lithium chloride, cholecystokinin and meal patterns: evidence that cholecystokinin suppresses meal size in rats without causing malaise. Appetite 8:221-227
Wiggins NL, McArthur C, McLean S, Boyle R (2003) Effects of two plant secondary metabolites, cineole and gallic acid, on nightly feeding patterns of the common brushtail possum. J Chem Ecol 29:1447-1464

Wiggins NL, Marsh KJ, Wallis IR, Foley WJ, McArthur C (2006) Sideroxylonal in Eucalyptus foliage influences foraging behaviour of an arboreal folivore. Oecologia 147:272-279 